\title{
Análise dos perfis epidemiológicos da leishmaniose visceral e da coinfecção \\ leishmaniose visceral-HIV no Piauí, 2007 a 2019
}

\author{
Analysis of the epidemiological profiles of visceral leishmaniasis and co-infection of visceral \\ leishmaniasis-HIV in Piauí, from 2007 to 2019 \\ Análisis de los perfiles epidemiológicos de leishmaniasis visceral y coinfección leishmaniasis \\ visceral-VIH en Piauí, 2007 a 2019
}

Recebido: 04/09/2021 | Revisado: 11/09/2021 | Aceito: 13/09/2021 | Publicado: 15/09/2021

Barbara Beatriz Lira da Silva ORCID: https://orcid.org/0000-0001-9166-6147

Universidade Estadual do Piauí, Brasil

E-mail: brbeatriz16@gmail.com

Ana Klara Rodrigues Alves

ORCID: https://orcid.org/0000-0002-1216-9386

Universidade Estadual do Piauí, Brasil

E-mail: klaraphb@outlook.com

Breno Carvalho de Almeida

ORCID: https://orcid.org/0000-0001-8736-9481

Universidade Estadual do Piauí, Brasil

E-mail:carvalhobreno019@gmail.com

Raquel de Brito Pereira

ORCID: https://orcid.org/0000-0002-4455-4449

Universidade Estadual do Piauí, Brasil

E-mail: raquelbritopp@gmail.com

Mariana Rios de Castro

ORCID: https://orcid.org/0000-0002-6261-4361

Universidade Estadual do Piauí, Brasil

E-mail: marianariosdecastro@gmail.com

Victória Andressa Paiva Pereira Santos

ORCID: https://orcid.org/0000-0002-1277-237X Universidade Estadual do Piauí, Brasil

E-mail: victoriandressa@hotmail.com

Maria Jacilene Alves Fontenele

ORCID: https://orcid.org/0000-0002-6671-639X Universidade Estadual do Piauí, Brasil E-mail: mariajacilene123@gmail.com

Larissa dos Santos Silva

ORCID: https://orcid.org/0000-0001-8485-4094 Universidade Estadual do Piauí, Brasil

E-mail: 1ssilva@aluno.uespi.br

Annderson Carneiro de Oliveira

ORCID: https://orcid.org/0000-0002-7363-5141 Mauricio de Nassau, Brasil

E-mail: annderson.ac@gmail.com

Fábio Dias Nogueira

ORCID: https://orcid.org/0000-0001-7303-1180 Faculdade de Ciências Humanas, Exatas e da Saúde do Piaú, Brasil

E-mail: fdnvaq@gmail.com

Francisco Robson de Oliveira Alves

ORCID: https://orcid.org/0000-0002-9854-6460 Universidade Federal do Piauí, Brasil

E-mail: robson_oliveira.fisio@outlook.com

Iris Lopes Veras

ORCID: https://orcid.org/0000-0002-7171-7207

Universidade de Fortaleza, Brasil e-mail: irislveras@outlook.com

Eugênia Mirza de Queiroz Ferreira Barboza da Silveira ORCID: https://orcid.org/0000-0002-3459-894X Universidade de Fortaleza, Brasil E-mail: eugenia.silveiraa@gmail.com 


\title{
Resumo
}

O presente estudo tem como objetivo analisar os perfis epidemiológicos da Leishmaniose Visceral (LV) e da coinfecção Leishmaniose Visceral-HIV (LV-HIV) no estado Piauí, no período de 2007 a 2019. O método de pesquisa utilizado foi o estudo epidemiológico descritivo, do tipo quantitativo, referente ao perfil epidemiológico dos casos de leishmaniose visceral e da coinfecção LV-HIV no Piauí do período de 2007 a 2019, notificados e confirmados no SINAN (Sistema de Informações de Agravos de Notificação). Dos 2.797 casos de leishmaniose visceral notificados foram descartados os casos "brancos/ignorados" (n=486) assinalados no subcampo 34 da ficha de notificação SINAN LV, totalizando uma amostra de 2.311 casos, dos quais 2.015 (87,2\%) apresentavam apenas LV e 296 (12,8\%) coinfecção LV-HIV. No Piauí, a LV é considerada um grave problema de saúde pública e foi constatado que os pacientes com leishmaniose visceral com e sem coinfecção HIV eram, em sua maioria, homens e procedentes da zona urbana, com idade de 1 a 4 anos para a LV e 20 a 39 anos para LV-HIV, com o principal método de confirmação laboratorial em ambos os grupos, tendo um bom percentual de cura (80\%), uma alta letalidade $(10,13 \%)$ e recidiva $(10 \%)$.

Palavras-chave: Leishmaniose visceral; Infecções por HIV; Epidemiologia.

\begin{abstract}
This study aims to analyze the epidemiological profiles of Visceral Leishmaniasis (VL) and Visceral LeishmaniasisHIV (VL-HIV) co-infection in the state of Piauí, from 2007 to 2019. The research method used was the descriptive epidemiological study, of the quantitative type, regarding the epidemiological profile of cases of visceral leishmaniasis and LV-HIV co-infection in Piauí from 2007 to 2019, notified and confirmed in the SINAN (Information System for Notifiable Diseases). Of the 2,797 cases of visceral leishmaniasis notified, the "white/ignored" cases $(n=486)$ marked in subfield 34 of the SINAN LV notification form were discarded, totaling a sample of 2,311 cases, of which 2015 (87.2\%) had only LV and 296 (12.8\%) LV-HIV co-infection. In Piauí, VL is considered a serious public health problem and it was found that patients with visceral leishmaniasis with and without HIV co-infection were mostly men and from urban areas, aged 1 to 4 years for VL and 20 to 39 years for LV-HIV, with the main method of laboratory confirmation in both groups, with a good percentage of cure (80\%), a high lethality (10.13\%) and recurrence (10\%).
\end{abstract}

Keywords: Visceral Leishmaniasis; HIV infections; Epidemiology.

\section{Resumen}

Este estudio tiene como objetivo analizar los perfiles epidemiológicos de la coinfección por Leishmaniasis Visceral (LV) y Leishmaniasis Visceral-VIH (LV-VIH) en el estado de Piauí, de 2007 a 2019 . El método de investigación utilizado fue el estudio epidemiológico descriptivo, de la tipo cuantitativo, referido al perfil epidemiológico de los casos de leishmaniasis visceral y coinfección VI-VIH en Piauí de 2007 a 2019, notificados y confirmados en el SINAN (Sistema de Información de Enfermedades Notificables). De los 2.797 casos de leishmaniasis visceral notificados, se descartaron los casos "blancos / ignorados" $(\mathrm{n}=486)$ marcados en el subcampo 34 del formulario de notificación SINAN LV, totalizando una muestra de 2.311 casos, de los cuales $2015(87,2 \%)$ Coinfección LV y 296 $(12,8 \%)$ LV-VIH. En Piauí, la LV se considera un problema de salud pública grave y se encontró que los pacientes con leishmaniasis visceral con y sin coinfección por VIH eran en su mayoría hombres y de áreas urbanas, de 1 a 4 años para LV y de 20 a 39 años para LV- VIH, con el principal método de confirmación de laboratorio en ambos grupos, con un buen porcentaje de curación $(80 \%)$, alta letalidad $(10,13 \%)$ y recurrencia $(10 \%)$.

Palabras clave: Leishmaniasis visceral; Infecciones por VIH; Epidemiología.

\section{Introdução}

A Leishmaniose Visceral (LV), também denominada calazar, é uma infecção causada por parasitos do gênero Leishmania (principalmente pelo complexo Leishmania donovani e Leishmania infantum), transmitidos no Brasil por meio da picada das fêmeas dos flebotomíneos da espécie Lutzomyia longipalpis (conhecidos popularmente como mosquitos-palha). Esses parasitos são protozoários heteroxênicos e intracelulares obrigatórios que afetam células do sistema fagocitário mononuclear de seus hospedeiros, tanto animais quanto o homem, sendo o cão o principal reservatório doméstico (Silva, 2018). 
Trata-se de uma doença crônica e sistêmica, caracterizada por febre de longa duração, perda de peso, astenia, adinamia, hepatoesplenomegalia e anemia, entre outros sintomas, que quando não tratada, pode evoluir para o óbito em mais de $90 \%$ dos casos. Seu diagnóstico é clínico, laboratorial e parasitológico, através de ferramentas sensíveis e específicas, enquanto o tratamento é disponibilizado gratuitamente pelo Sistema Único de Saúde (SUS), através do fármaco de primeira escolha Antimoniato de N-metil glucamina (exceto em algumas situações, nas quais se recomenda o uso da anfotericina B) com cerca de 100\% dos pacientes evoluindo para cura clínica quando tratados oportunamente (Brasil, 2016).

Aspectos relacionados à sua incidência e alta letalidade (principalmente entre indivíduos não tratados, pessoas em situação de vulnerabilidade social, com habitações precárias, crianças desnutridas e indivíduos portadores da infecção pelo Vírus da Imunodeficiência Humana (HIV) contribuem para que a Leishmaniose visceral esteja incluída entre as seis doenças endêmicas mais importantes no mundo (Alves \& Fonseca, 2018).

Segundo a Organização Mundial da Saúde (OMS), a LV é uma das doenças tropicais mais negligenciadas mundialmente, com estimativas de aproximadamente 50 a 90 mil casos novos por ano, sendo que mais de $90 \%$ destes ocorrem em sete países: Brasil, Etiópia, Índia, Quênia, Somália, Sudão do Sul e Sudão (Who, 2016).

No Brasil, embora seja uma doença que teve seu início nas áreas rurais, principalmente do Nordeste, a partir da década de 1980, essa zoonose expandiu-se e experimentou uma mudança epidemiológica importante, passando em algumas regiões a ter um caráter essencialmente urbano e periurbano, constituindo um grave problema de saúde pública e de notificação compulsória (Santos, Rodrigues, Nascimento, Rodrigues \& Góes, 2018).

De acordo com o Ministério da Saúde, em 19 anos (1984-2002) foram notificados 48.455 casos de LV, dos quais aproximadamente 66\% ocorreram na Bahia, Ceará, Maranhão e Piauí. No período de 2003 a 2012, a média anual de LV foi de 3.565 casos e a incidência de 1,9 caso/100.000 hab. No mesmo período, a letalidade média foi de $6,9 \%$, atingindo os maiores percentuais nos anos de 2003 (8,5\%) e 2004 (8,2\%) (Brasil, 2016).

Mudanças recentes nas tendências epidemiológicas apontam que nos últimos anos houve claramente uma elevada incidência de casos de LV em áreas de alta transmissão do HIV (coinfecção LV-HIV) o que permitiu a identificação do referido vírus como um dos desafios emergentes para o controle da LV, devido essa sobreposição das patologias (Van Griensven, Carrillo, López-vélez, Lynen \& Moreno, 2014; Alemayehu, 2017).

O diagnóstico de LV depende de métodos clínicos e de testes laboratoriais. No entanto, em pacientes infectados pelo HIV, a sensibilidade da sorologia é muito baixa, e o diagnóstico parasitológico é o que apresenta a mais alta especificidade, sendo necessário um examinador experiente para o diagnóstico (Who, 2016; Martins-melo, Lima, Alencar, Ramos Jr \& Heukelbach, 2014).

Pacientes infectados com HIV são mais propensos a desenvolver sintomatologia de LV, seja em decorrência da reativação de uma infecção latente de Leishmania (adquirida antes do HIV) ou devido as manifestações clínicas associadas à Leishmania tornarem-se mais evidentes após infecção pelo HIV. Portanto, a LV é considerada uma infecção oportunista em pacientes com HIV, muitas vezes apresentando-se com características clínicas atípicas, como o acometimento dos tratos gastrointestinal e respiratório encontradas em pacientes com baixa contagem de linfócitos CD4+ ( $<200$ células $\left./ \mathrm{mm}^{3}\right)$ ou parasitas isolados em lugares incomuns (Monge-maillo, Norman, Cruz, Alvar \& López-vélez, 2014; Mahajan et al., 2015; Lindoso, Cunha, Queiroz \& Moreira, 2016).

A Leishmania desenvolveu estratégias para sobreviver e multiplicar-se dentro de macrófagos em pacientes com HIV, gerando a possibilidade de resistência aos medicamentos e falha do tratamento. Apesar dos dados escassos sobre a magnitude e os fracos resultados do tratamento da coinfecção LV-HIV, entende-se que um dos principais desafios atuais é o desenvolvimento de uma terapia eficaz da droga que não só resolva o primeiro episódio de LV, mas também impeça recaídas. Até à data, a anfotericina $\mathrm{B}$ e suas formulações lipídicas, antimoniais pentavalentes, paromomicina, e miltefosina, 
demonstraram eficácia contra LV em pacientes imunocompetentes. No entanto, há pouca evidência da sua eficácia em pacientes coinfectados LV-HIV (Monge-maillo \& López-vélez, 2016).

Em 2015, dos 257 casos de coinfecção LV-HIV notificados na América Latina, 244 ocorreram no Brasil, 12 no Paraguai e um caso na Venezuela, representando um aumento de 6\% dos casos em relação à 2014 (Who, 2016). Há de se ressaltar que embora as estatísticas apontem que o Brasil é o país com maior número de casos notificados de LV (mais de 96\%) e da coinfecção LV-HIV, esses dados devem ser interpretados com cautela, já que as subnotificações também ocorrem de modo expressivo (Lindoso et al., 2016; Silva, 2018).

Os pacientes coinfectados com LV-HIV apresentam maior risco de recidivas, maior letalidade e mortalidade, o que pode ser atribuído à complicações da LV, incluindo resistência à terapia medicamentosa, a ocorrência de outras infecções oportunistas e comorbidades, além de atraso no diagnóstico (Silva, 2018). Ao mesmo tempo em que as pessoas infectadas pelo HIV são particularmente vulneráveis à LV - o risco de desenvolvimento de LV é 100-2.300 vezes superior nos doentes infectados pelo HIV do que em indivíduos soronegativos - a Leishmania acelera a replicação do HIV e a progressão da infecção viral para a AIDS (Goswami et al., 2017; Lindoso, Moreira, Cunha \& Queiroz, 2018).

Nesse contexto, o presente estudo analisou os perfis epidemiológicos da Leishmaniose Visceral (LV) e da coinfecção Leishmaniose Visceral-HIV (LV-HIV) no estado Piauí, no período de 2007 a 2019.

\section{Metodologia}

Trata-se de um estudo epidemiológico descritivo, do tipo quantitativo, onde se adotou os fundamentos metodológicos da epidemiologia que conforme Rouquayrol (1994) é a elaboração do problema epidemiológico, passando por fontes geradoras do mesmo, raciocínio epidemiológico, variáveis e hipóteses epidemiológicas até a arquitetura da investigação. A pesquisa aborda o perfil epidemiológico dos casos de leishmaniose visceral e da coinfecção LV-HIV no Piauí do período de 2007 a 2019. A população do estudo foi composta por todos os casos de LV notificados e confirmados no SINAN (Sistema de Informações de Agravos de Notificação). no estado do Piauí do ano de 2007 a 2019. O banco de dados utilizado foi aquele disponibilizado pelo Departamento de Informática do Sistema Único de Saúde (DATASUS) da Secretaria de Vigilância em Saúde/ Ministério da Saúde. Esse banco de dados possui a identificações de informações demográficas, clínicas e epidemiológicas dos pacientes notificados do SINAN LV.

A partir da ficha de notificação do SINAN LV, utilizou-se o subcampo 34 ("Coinfecção HIV") para mensuração, dentro da população, dos casos de LV-HIV ocorridos no estado do Piauí durante o período proposto no estudo.

Foram utilizados na pesquisa dados secundários, obtidos no banco de dados do SINAN, os quais são disponibilizados por meio eletrônico no portal do DATASUS do Ministério da Saúde.

A coleta de dados ocorreu entre janeiro e fevereiro de 2021. As variáveis utilizadas nesse estudo foram: ano de notificação, sexo, faixa etária, zona de residência (urbana, rural e periurbana), tipo de entrada (casos novos, recidivas e transferência), critério de confirmação (laboratorial ou clínico-epidemiológico), diagnóstico parasitológico e de imunoflourescência indireta, e evolução (cura, abandono, óbito por LV, óbito por outra causa e transferência). Para subsidiar a análise dos dados, todas as informações coletadas foram reunidas e posteriormente tabuladas, em planilhas do Microsoft Excel® para realização da análise descritiva e apresentada por meio de tabelas.

Foram incluídos no estudo todos os casos notificados no período de 2007 a 2019 excluídos do estudo casos cujo subcampo 34 (Coinfecção HIV) da ficha de notificação do SINAN LV não estavam preenchidos (em branco) apresentaram alguma inconsistência ou ausência de alguma informação das variáveis utilizadas na pesquisa. 
No presente estudo foram utilizados dados do tipo secundário, obtidos através do sítio eletrônico do DATASUS na internet, sendo esse de domínio público. Diante disso, torna-se dispensável a apreciação por um Comitê de Ética em Pesquisa (CEP).

Uma das limitações deste estudo decorreu do fato de ser retrospectivo e depender da qualidade dos registros.

\section{Resultados}

Dos 2.797 casos de leishmaniose visceral notificados foram descartados os casos "brancos/ignorados" (n=486) assinalados no subcampo 34 (coinfecção HIV) da ficha de notificação SINAN LV, totalizando uma amostra de 2.311 casos, dos quais $2.015(87,2 \%)$ apresentavam apenas leishmaniose visceral e 296 (12,8\%) coinfecção LV-HIV (Tabela 1).

Tabela 1. Casos confirmados de LV segundo ano de notificação e Coinfecção HIV, no período de 2007-2019, Piauí, Brasil.

\begin{tabular}{llll}
\hline Ano de notificação & Coinfecção LV-HIV & LV & Total* \\
& n $(\%)$ & \multicolumn{1}{l}{$(\%)$} & 178 \\
\hline 2007 & - & $178(8,83 \%)$ & 158 \\
2008 & $18(6 \%)$ & $140(6,94 \%)$ & 147 \\
2009 & $22(7,43 \%)$ & $125(6,20 \%)$ & 139 \\
2010 & $17(5,74 \%)$ & $122(6 \%)$ & 179 \\
2011 & $18(6 \%)$ & $161(7,99 \%)$ & 170 \\
2012 & $43(14,52 \%)$ & $127(6,3 \%)$ & 187 \\
2013 & $18(6 \%)$ & $169(8,38 \%)$ & 256 \\
2014 & $26(8,78 \%)$ & $230(11,41 \%)$ & 218 \\
2015 & $29(9,79 \%)$ & $189(9,37 \%)$ & 163 \\
2016 & $28(9,45 \%)$ & $135(6,69 \%)$ & 203 \\
2017 & $33(11,14 \%)$ & $170(8,43 \%)$ & 172 \\
2018 & $20(6,75 \%)$ & $152(7,54 \%)$ & 141 \\
2019 & $24(8,10 \%)$ & $117(5,80 \%)$ & $2311^{*}$ \\
\hline \hline Total & 296 & 2015 & \\
\hline
\end{tabular}

*Foram excluídos 486 casos ignorados/brancos.

Fonte: Ministério da Saúde/ SINAN (2021).

A análise da frequência de casos por ano, evidenciou que 2014 apresentou a maior frequência de notificações sobre LV ( $\mathrm{n}=230 ; 11,41 \%)$ assim como da totalidade geral de casos ( $\mathrm{LV}+\mathrm{LV} / \mathrm{HIV})(\mathrm{n}=256)$. Por sua vez, o ano com maior frequência de casos de LV-HIV foi 2012 ( $\mathrm{n}=43 ; 14,52 \%)$. Ao longo do período analisado observou-se oscilações nas frequências tendo havido redução a partir de 2018 (Tabela 1).

Em relação à zona de residência dos pacientes infectados, observou-se que a maioria dos casos ocorreu em zona urbana ( $\mathrm{n}=1.588)$, e houve predomínio do sexo masculino em ambos os grupos, na infecção por LV com 1.328 (66\%) casos e 241 (81\%) casos entre aqueles da coinfeção LV-HIV. A média geral da razão de sexos no período analisado foi de 2,11 casos masculinos para cada caso feminino. Quanto ao critério de confirmação da patologia em ambos os grupos prevaleceu o diagnóstico laboratorial, realizado em 1.762 (87,44\%) casos de LV e 273 (92\%) daqueles com LV/ HIV (Tabela 2). 
Tabela 2. Casos confirmados de LV e Coinfecção LV-HIV segundo, Zona de residência, Sexo, e confirmação dos casos confirmados, no período de 2007-2019, Piauí, Brasil.

\begin{tabular}{|c|c|c|c|}
\hline \multirow[t]{2}{*}{ Características } & \multirow{2}{*}{$\begin{array}{l}\text { Coinfecção LV-HIV } \\
\text { n }(\%)\end{array}$} & \multirow{2}{*}{$\begin{array}{l}\mathrm{LV} \\
\mathrm{n} \quad(\%)\end{array}$} & \multirow{2}{*}{$\begin{array}{l}\text { Total* } \\
\text { N }\end{array}$} \\
\hline & & & \\
\hline \multicolumn{4}{|l|}{ Zona de } \\
\hline \multicolumn{4}{|l|}{ Residência } \\
\hline Zona Urbana & $255(86 \%)$ & $1337(68,14 \%)$ & 1.558 \\
\hline Zona Rural & $41(14 \%)$ & $619(31,55 \%)$ & 660 \\
\hline Periurbana & 0 & $6(0,46 \%)$ & 6 \\
\hline \multirow[t]{5}{*}{ Total } & 296 & 2015 & $2311 *$ \\
\hline & $241(81 \%)$ & $1.328(66 \%)$ & 1.569 \\
\hline & $55(19 \%)$ & $687(34 \%)$ & 742 \\
\hline & 4,38 & 1,93 & 2,11 \\
\hline & 296 & 2.015 & $2311 *$ \\
\hline
\end{tabular}

\begin{tabular}{llll}
\hline Laboratorial & $273(92 \%)$ & $1762(87,44 \%)$ & 2.035 \\
Clínico-epidemiológico & $23(8 \%)$ & $253(12,56 \%)$ & 276 \\
\hline Total & 296 & 2.015 & $2311^{*}$ \\
\hline
\end{tabular}

Fonte: Ministério da Saúde/ SINAN (2021).

*Foram excluídos 486 casos ignorados/brancos.

A faixa etária com o maior número de casos notificados de LV foi a de 1 -4 anos (n=543; 27\%) e daqueles com LVHIV foi de 20-39 anos ( $\mathrm{n}=163 ; 55 \%)$ (Tabela 3$)$. 
Tabela 3. Casos confirmados de LV e Coinfecção LV-HIV segundo Faixa Etária, no período de 2007-2019, Piauí, Brasil.

\begin{tabular}{llll}
\hline Faixa Etária & Coinfecção LV-HIV & LV & Total \\
& $n(\%)$ & n & N \\
& & $240(12 \%)$ & 243 \\
\hline$<1$ ano & $3(1 \%)$ & $543(27 \%)$ & 551 \\
$1-4$ anos & $5(2 \%)$ & $163(8 \%)$ & 173 \\
$5-9$ anos & $5(2 \%)$ & $83(4 \%)$ & 85 \\
$10-14$ anos & $2(1 \%)$ & $93(5 \%)$ & 99 \\
$15-19$ anos & $6(2 \%)$ & $443(22 \%)$ & 609 \\
$20-39$ anos & $163(55 \%)$ & $296(15 \%)$ & 395 \\
$40-59$ anos & $99(33 \%)$ & $42(2 \%)$ & 49 \\
$60-64$ anos & $7(2 \%)$ & $33(2 \%)$ & 37 \\
$65-69$ anos & $4(1 \%)$ & $50(2 \%)$ & 52 \\
$70-79$ anos & $2(1 \%)$ & $18(1 \%)$ & 18 \\
80 e + & - & 2015 & $2311^{*}$ \\
\hline \hline Total
\end{tabular}

*Foram excluídos 486 casos ignorados/brancos.

Fonte: Ministério da Saúde/ SINAN (2021).

O principal método diagnóstico de confirmação da LV $(n=1.106 ; 54,9 \%)$ e da coinfecção $(n=214 ; 72,2 \%)$ foi o parasitológico. Outro método diagnóstico observado foi a imunofluorescência indireta obteve confirmação de 21,78\% (n=439) para LV e 11,14\% $(n=33)$ para LV-HIV, mas é preciso destacar a elevada frequência de exames não realizados entre pacientes com LV $(67,14 \%)$ e coinfecção LV-HIV (80,06\%) (Tabela 4).

Tabela 4. Casos confirmados de LV e Coinfecção LV-HIV segundo Diagnóstico parasitológico, no período de 2007-2019, Piauí, Brasil.

\begin{tabular}{llll}
\hline Características & $\begin{array}{l}\text { Coinfecção LV-HIV } \\
\text { n }(\%)\end{array}$ & Total* \\
& & & N \\
\hline Diagnóstico Parasitológico & & $1.106(54,9 \%)$ & 1.320 \\
Positivo & $214(72,2 \%)$ & 448 \\
Negativo & $41(13,9 \%)$ & $407(20,2 \%)$ & 543 \\
Não realizado & $41(13,9 \%)$ & $502(24,9 \%)$ & 2.205 \\
\hline Total & 296 & 2.015 & 472 \\
\hline Imunofluorescência Indireta & & $439(21,78 \%)$ & 249 \\
Positivo & $33(11,14 \%)$ & $223(11,06 \%)$ & 1.590 \\
Negativo & $26(8,8 \%)$ & $1.353(67,14 \%)$ & 2.311 \\
Não realizado & $237(80,06 \%)$ & 2.015 & \\
\hline Total & 296 & & \\
\hline
\end{tabular}

*Foram excluídos 592 casos ignorados/brancos do Diagnóstico Parasitológico e 486 casos ignorados/brancos do Diagnóstico por Imunofluorescência Indireta.

Fonte: Ministério da Saúde/ SINAN (2021). 
Em relação aos tipos de entradas foram observados uma totalidade de 2.183 casos novos (94\%), 109 (4\%) recidivas e 19 (1\%) transferências. A taxa de cura foi de $80 \%(n=1.032)$ e $84 \%(n=182)$ entre aqueles com LV e coinfectados respectivamente. Houve um total 134 óbitos por LV (9\%) e 24 (1,65\%) por outras causas, onde a coinfecção, quando comparada somente com o LV, possui uma taxa maior em ambos os óbitos $13 \%$ e $6 \%$, respectivamente (Tabela 5).

Tabela 5. Casos confirmados de LV e Coinfecção LV-HIV segundo Tipo de entrada e Evolução, no período de 2007-2019, Piauí, Brasil.

\begin{tabular}{llll}
\hline Características & Coinfecção LV-HIV & Total \\
& $\mathrm{n}(\%)$ & $\mathrm{N}$ & $\mathrm{N}$ \\
\hline Tipo de entrada & & & \\
& & & \\
Casos Novos & $263(88 \%)$ & $1.920(95 \%)$ & 2.183 \\
Recidivas & $31(10 \%)$ & $78(4 \%)$ & 109 \\
Transferências & $2(2 \%)$ & $17(1 \%)$ & 19 \\
\hline Total & 296 & 2.015 & $2311^{*}$ \\
\hline & & & 1.214 \\
& $182(80 \%)$ & $1.032(84 \%)$ & 8 \\
& $1(0,5 \%)$ & $7(0,6 \%)$ & 134 \\
& $30(13 \%)$ & $104(8,5 \%)$ & 24 \\
\hline $14(6 \%)$ & $10(0,9 \%)$ & 71 \\
\hline
\end{tabular}

*Foram excluídos 486 casos ignorados/brancos com relação a coinfecção com HIV e 994 casos ignorados/ brancos com relação a variável evolução.

Fonte: Ministério da Saúde/ SINAN (2021).

\section{Discussão}

No Piauí, a LV é considerada um grave problema de saúde pública, e muitos fatores contribuíram para a alta incidência de LV desde a criação do estado: desmatamento com fins lucrativos, criação de animais em quintais (principalmente galinhas e cães), intenso fluxo migratório e falta de saneamento básico. A disseminação de portadores da doença pode explicar a oscilação dos casos durante o passar dos anos no estado (Brasil, 2014).

O número de casos de LV entre os indivíduos infectados pelo HIV aumentou substancialmente, o que confirma a premissa que o risco de desenvolver LV parece ser consideravelmente maior na população infectada pelo HIV (Henn et al., 2018). O presente estudo mostra uma variação de números durante os anos, sendo ainda relevante observar que existe uma grande quantidade de subnotificações. Apesar do alto número de casos relatados de LV relacionada ao HIV, certos aspectos de sua epidemiologia, características clínicas e manejo permanecem desconhecidos (Hurissa et al., 2010).

No presente estudo os municípios que registraram os maiores contingentes de casos foram Teresina, Parnaíba e Miguel Alves, corroborando com achados encontrados por Batista et al (2014).

A maioria dos casos eram de áreas urbanas, o que é consistente com a tendência de urbanização de LV observada em muitos países, incluindo o Brasil, desde o final da década de 1990. A pobreza e as escassas temporadas de chuva levam a uma 
intensa migração interna das áreas rurais para as urbanas constituindo fatores importantes que podem explicar esta ocorrência (Tavora, Nogueira \& Gomes, 2015).

A LV geralmente começa durante a infância, enquanto para aqueles coinfectados com LV-HIV geralmente afeta adultos jovens, conforme descrito por Coutinho et al. (2017) Viana et al. (2017) O presente estudo confirma esses achados: a maioria dos casos de coinfecção ocorreu na faixa etária de 20-39 anos e de LV de 1-4 anos. Isso é observado não só no Piauí, como, também, em outros Estados, onde o acometimento em crianças acontece principalmente porque o sistema imunológico não está totalmente desenvolvido. Esse acometimento é agravado por carência nutricional e pelo fato de que as crianças estarem mais expostas ao vetor no ambiente peridomiciliar (Lemos et al., 2019).

O sexo masculino responde pela maior percentagem de casos registrados no período de avaliação do presente estudo, tanto na LV (66\%), como na LV-HIV (81\%). A predominância de LV nesse sexo está relacionada a uma maior exposição ao vetor em regiões agrícolas, enquanto as mulheres estão, em geral, menos expostas e, na maior parte das vezes, ocupando ambientes intra e peridomiciliares (Luz et al., 2018).

O critério para confirmação da LV e LV-HIV mais utilizado no Piauí, no período em estudo, foi o laboratorial/parasitológico. O critério clínico de confirmação mais confiável para LV é o laboratorial, no qual é possível identificar o parasito por meio de exames específicos e inespecíficos. Independentemente se o caso é LV ou coinfecção LVHIV, o diagnóstico parasitológico continua a ser uma ferramenta de confirmação por causa de sua alta especificidade (Brasil, 2011).

Em indivíduos infectados com LV-HIV os métodos de diagnóstico parasitológico devem ser preferidos. Em teoria, métodos para detectar antígenos funcionariam melhor no diagnóstico de LV em pacientes infectados pelo HIV, pois poderiam estar relacionados à carga parasitária, porém foi visto que a sensibilidade é mais baixa em indivíduos coinfectados, demonstrando que os mesmos são menos confiáveis nesses pacientes (Lindoso et al., 2018).

A evolução da LV e LV-HIV apresentou valores significativos para a cura clínica $80 \%$ e $84 \%$, respectivamente, tendo apenas $0,55 \%$ de abandono total. O critério de cura, para essa patologia, envolve o acompanhamento do doente por 12 meses. Os principais critérios de cura são clínicos, sendo eles: redução dos órgãos hipertrofiados (baço e fígado), ausência de febre, regulação de índices hematológicos, ganho gradativo de peso, presença de eusinofilia, durante ou no final do tratamento. No entanto, as notificações de abandono dos casos que não completaram o tratamento ou que não compareceram para avaliação clínica no dia previsto devem ser acompanhadas pelo serviço de saúde, com intuito de acompanhar sua gravidade (Brasil, 2011).

Para os coinfectados, a proporção de recidivas foi duas vezes maior do que para os não-coinfectados. A tendência à recidiva é uma das características mais observadas nos estudos de coinfecção de LV com HIV (Luz et al., 2018). Os números de óbitos foram maiores em indivíduos coinfectados, tanto por LV (13\%) como por outras causas (6\%). Quando a LV ocorre em indivíduos infectados pelo HIV, o prognóstico é tipicamente ruim e a taxa de mortalidade é alta. As taxas de letalidade e a recidiva refletem a importância da deteç̧ão e tratamento precoce do HIV em pacientes com LV, a fim de iniciar o tratamento adequado e prevenir a mortalidade (Guedes et al., 2021).

A letalidade foi de 10,13\% nos casos coinfectados, duas vezes maior do que entre não coinfectados. Vários autores descrevem uma maior taxa de letalidade entre pacientes coinfectados com LV-HIV, e a causa de morte dos pacientes coinfectados sendo outras condições associadas à AIDS, como doenças oportunistas e complicações terapêuticas (Sousagomes, Elkhoury \& Pelissari, 2011; Batista et al., 2014; Távora et al., 2015). Este resultado mostra que é necessário estabelecer medidas de controle dessa doença no Piaú, uma vez que o Ministério da Saúde (Brasil, 2014) preconiza que a taxa de letalidade deve ser inferior a $5 \%$. 
A relação entre a coinfecção LV-HIV e a letalidade de LV não está bem elucidada, pois não se sabe se a gravidade se deve à infecção pelo HIV ou consequentes infecções oportunistas. Tomados em conjunto, esses achados reforçam a ideia de que a letalidade relacionada à LV é uma grande preocupação atual e ressaltam a necessidade de melhorar o diagnóstico e o manejo dos pacientes, incluindo o rastreamento da infecção pelo HIV (Sousa-gomes, Romero, \& Werneck, 2017).

Todo óbito de LV deve ser investigado para saber as causas determinantes; a busca e o monitoramento devem ser realizados por intermédio de notificações no SINAN e no Sistema de Mortalidade (SIM). Depois da coleta de dados, os profissionais envolvidos devem examinar os casos, procurando medidas que corrijam as eventuais falhas, com o objetivo de diminuir a letalidade dessa infecção.

Devido ao aumento do risco de recaídas e ao mau prognóstico, é importante para pessoas HIV-positivas, particularmente aquelas que vivem em áreas endêmicas de LV, saber sobre uma infecção de Leishmania anterior. A identificação de novos casos que possam delimitar a doença ativa, sugerir cura e prever recidivas é urgente. Portanto, pode ser um desafio distinguir os casos de LV ativos de outra infecção oportunista, e políticas públicas de saúde para LV podem ajudar os profissionais de saúde a fazer diagnósticos mais precisos e, consequentemente, evitar tratamentos desnecessários (Guedes et al., 2021; Brasil, 2015).

\section{Conclusão}

No estado do Piauí foi constatado que os pacientes com leishmaniose visceral com e sem coinfecção HIV eram, em sua maioria, homens e procedentes da zona urbana, com idade de 1 a 4 anos para a LV e 20 a 39 anos para LV-HIV, com o principal método de confirmação laboratorial em ambos os grupos, tendo um bom percentual de cura, uma alta letalidade e recidiva.

A situação epidemiológica da coinfecção LV-HIV no Piauí evidenciada por este estudo é preocupante. O crescente número de casos de coinfecção é um desafio para o sistema de saúde, principalmente no que se refere ao impacto na letalidade e recidiva de pacientes com LV. Nesse sentido, intervenções destinadas a reduzir a incidência e a deteç̧ão precoce da infecção pelo HIV, bem como o início do tratamento específico, são fundamentais para reduzir a incidência da coinfecção.

Além disso, o estudo confirma a necessidade dos pacientes com suspeita ou confirmação de LV serem testados para HIV; além de incluir LV no diagnóstico diferencial de patologias que envolvem pacientes infectados pelo HIV; e implementar profilaxia secundária em pacientes que tiveram LV, para prevenir a recorrência e mau prognóstico. No entanto, são necessários mais estudos que demonstrem o impacto da letalidade e recidiva em pacientes com LV-HIV.

\section{Referências}

Alemayehu, M. (2017). Perceived quality of life among Visceral Leishmaniasis and HIV coinfected migrant male-workers in Northwest Ethiopia: a qualitative study. BMC Public Health. 17(204), 1-8.

Alves, W. A. \& Fonseca, D. S. (2018). Leishmaniose visceral humana: perfil clínico-epidemiológico na região leste de Minas Gerais. J. Health Biol Sci, 6(2), 133-139.

Batista, F. M. A., Machado, F. F. O. A., Silva, J. M. O., Mittmann, J., Barja, P. R. \& Simioni, A. R. (2014). Leishmaniose: perfil epidemiológico dos casos notificados no estado do Piauí entre 2007 e 2011. Rev Univap. 20(35).

Brasil, Ministério da Saúde (2011). Secretaria de Vigilância em Saúde. Departamento de Vigilância Epidemiológica. Manual de recomendação para diagnóstico, tratamento e acompanhamento de pacientes com a coinfecção Leishmania -HIV. 1ª edição. Brasília: MS; 2011.

Brasil, Ministério da Saúde (2014). Secretaria de Vigilância em Saúde, Departamento de Vigilância Epidemiológica. Manual de vigilância e controle da leishmaniose visceral. Brasília, 2014.

Brasil, Ministério da Saúde (2015). Secretaria de Vigilância em Saúde. Manual de recomendação para diagnóstico, tratamento e acompanhamento de pacientes com a coinfecção leishmania-HIV.1.ed. Brasília. 2015. 
Brasil, Ministério da Saúde (2016). Secretaria de Vigilância em Saúde, Departamento de Vigilância Epidemiológica. Guia de vigilância em saúde: leishmaniose visceral. Brasília, 2016.

Coutinho, J. V., Santos, F. S., Ribeiro, R. S. P., Oliveira, I. B. B, Dantas, V. B., Santos, A. B. F. S \& Tauhata, J. R. (2017). Leishmaniose visceral e coinfecção leishmaniose-HIV: estudo comparativo. Rev Soc Bras Med Trop. 50, 670-674.

Goswami, R. P., Goswami, R. P., Basu, Y., Ray, Y., Rahman, M. \& Tripathi, S. K. (2017). Protective Efficacy of Secondary Prophylaxis against Visceral Leishmaniasis in Human Immunodeficiency Virus Coinfected Patients over the Past 10 Years in Eastern India. Am. J. Trop. Med. Hyg. 96(2), $285-291$.

Guedes, D. L., Justo, A. M., Barbosa Júnior, W. L., Silva, E. D., ,Aquino, S. R., Lima Júnior, M. S. C., Montarroyos, U., Bezerra, G. S. N., Vieira, A. V. B., Pereira, V. R. A. \& Medeiros, Z. M. (2021). Asymptomatic Leishmania infection in HIV-positive outpatients on antiretroviral therapy in Pernambuco, Brazil. PLoS Negl Trop Dis.15(1), e0009067.

Henn, G. A. L., Ramos Júnior, A. N., Colares, J. K. B., Mendes, L. P., Silveira, J. G. C., Lima, A. A. F., Aires, B. P. \& Façanha, M. C. (2018). Is Visceral Leishmaniasis the same in HIV-coinfected adults? Braz J Infect Dis. 22(2), 92-98.

Hurissa, Z., Gebre-Silassie, S., Hailu, W., Tefera, T., Lalloo, D. G., Cuevas, L. E. \& Hailu (2010). Clinical characteristics and treatment outcome of patients with visceral leishmaniasis and HIV co-infection in northwest Ethiopia. Trop Med Int Health. 15(7), 848-855.

Lemos, M. H. S., Silva, W. C., Gomes, F. C. S., Lages, L. P., Costa, J. O., Assis júnior, J. D. P., Teixeira, D. R. A. \& Antonio, F. (2019). Epidemiologia das leishmanioses no estado do Piauí. Braz. J. Surg. Clin. Res. 25(2), 53-57.

Lindoso, J. A., Cunha, M. A., Queiroz, I. T., \& Moreira, C. H. V. (2016). HIV Leishmaniasis co-infection: current challenges. HIV AIDS (Auckl), 8, 147-156.

Lindoso, J. A. L., Moreira, C. H. V., Cunha, M. A. \& Queiroz, I. T. (2018). Visceral leishmaniasis and HIV co-infection: current perspectives. HIV AIDS (Auckl), 10, 193-201.

Luz, J. G. G. Naves, D. B., Carvalho, A. G. C., Meira, G. A., Dias, J. V. L. \& Fontes, C. J. F. (2018). Visceral leishmaniasis in a Brazilian endemic area: an overview of occurrence, HIV coinfection and lethality. Rev Inst Med Trop, 60, e12.

Mahajan, R., Das, P., Isaakidis, P., Sunyoto, T., Sagili, K. D., Lima, M. A., Mitra, G., Kumar, D., Pandey, K., Van geertruyden, J., Boelaert, M. \& Burza, S. (2015). Combination Treatment for Visceral Leishmaniasis Patients Coinfected with Human Immunodeficiency Virus in India. Clin Infect Dis. 61(8), 12551262.

Martins-melo, F. R., Lima, M. S., Alencar, C. H., Ramos Jr, A. N. \& Heukelbach, J. (2014). Epidemiological patterns of mortality due to visceral leishmaniasis and HIV/AIDS co-infection in Brazil, 2000-2011. Trans R Soc Trop Med Hyg. 108, 338-347.

Mastroianni, A., Gaibani, P., Rossini, G., Vocale, C., Re, M. C., Ravaglia, G., Vittorio Sambri, V. \& Varani, S. (2018). Two cases of relapsed HIV-associated visceral leishmaniasis successfully treated with combination therapy. AIDS Res Ther. 15(27), 1-4.

Monge-maillo, B., Norman, F. F., Cruz, I., Alvar, J. \& López-Vélez, R. (2014). Visceral Leishmaniasis and HIV Coinfection in the Mediterranean Region. PLoS Negl Trop Dis. 8, e3021.

Monge-maillo, B. \& López-vélez, R. (2016). Treatment Options for Visceral Leishmaniasis and HIV Coinfection. AIDS Ver. 18(1), $32-43$.

Santos, M. A., Rodrigues, S. L. C., Nascimento, A. L. F., Rodrigues, J. S. \& Góes, M. A. O. (2018). Leishmaniose Visceral: Características clínicoepidemiológicas de casos e óbitos no estado de Sergipe. Rev. Epidemiol. Controle Infec. Santa Cruz do Sul. 8(4), 428-434.

Silva, L. R. M. (2018). Distribuição espacial da coinfecção leishmaniose visceral-hiv em Tersisa/PI, 2006 a 2015. [Tese]. Rio de Janeiro: Programa de Pósgraduação em saúde coletiva (Mestrado) - Universidade do Estado do Rio de Janeiro, Instituto de Medicina Social.

Sousa-gomes, M. L., Elkhoury, A. N. S. M. \& Pelissari, D. M. (2011). Coinfecção Leishmanial / HIV no Brasil: aspectos epidemiológicos, clínicos e laboratoriais. Epidemiol Serv Saúde. 20(4), 519-26.

Sousa-gomes, M. L., Romero, G. A. S. \& Werneck, G. L. (2017). Visceral leishmaniasis and HIV/AIDS in Brazil: Are we aware enough? PLoS Negl Trop Dis. 11 (9), e0005772.

Távora, L. G., Nogueira, M. B. \& Gomes, S. T. (2015). Coinfecção Leishmaniose Visceral / HIV no Nordeste do Brasil: avaliação do desfecho. Braz J Infect Dis. 19, 651-656.

Van griensven, J., Carrillo, E., López-Vélez, R., Lynen, L. \& Moreno, J. (2014). Leishmaniasis in immunosuppressed individuals. Clin Microbiol Infect, 20(4), 286-299, 2014.

Van griensven, J., Mengesha, B., Mekonnen, T., Fikre, H.,Takele, Y., Adem, E., Mohammed, R., Ritmeijer, K., Vogt, F., Adriaensen, W. \& Diro, E. (2018). Leishmania Antigenuria to Predict Initial Treatment Failure and Relapse in Visceral Leishmaniasis/HIV Coinfected Patients: An Exploratory Study Nested Within a Clinical Trial in Ethiopia. Front Cell Infect Microbiol. 8(94).

Viana, G. M. C., Silva, M. A. C. N., Garcia, J. V. S., Guimarães, H. D. G., Arcos Júnior, G. F., Santos, A. V. A., Paixão, P. V., Nascimento, M. D. S. B \& Galvão, C. S. (2017). Perfil epidemiológico de pacientes co-infectados com leishmaniose visceral e HIV / AIDS no Nordeste, Brasil. Rev. Soc. Bras. Med. Trop. 50(5).

Who. (2016). Weekly epidemiological record Relevé épidemiologique hebdomadaire. 21(83), 421-428. 